\title{
ISOMETRIES AND SPECTRA OF MULTIPLICATION OPERATORS ON THE BLOCH SPACE
}

\author{
ROBERT F. ALLEN and FLAVIA COLONNA ${ }^{\bowtie}$
}

(Received 10 March 2008)

\begin{abstract}
In this paper, we establish bounds on the norm of multiplication operators on the Bloch space of the unit disk via weighted composition operators. In doing so, we characterize the isometric multiplication operators to be precisely those induced by constant functions of modulus 1 . We then describe the spectrum of the multiplication operators in terms of the range of the symbol. Lastly, we identify the isometries and spectra of a particular class of weighted composition operators on the Bloch space.
\end{abstract}

2000 Mathematics subject classification: primary 47B38; secondary 30D45.

Keywords and phrases: multiplication operators, Bloch space, little Bloch space.

\section{Introduction}

Let $\mathbb{D}$ denote the open unit disk in the complex plane. An analytic function $f$ on $\mathbb{D}$ is said to be Bloch if

$$
\beta_{f}=\sup _{z \in \mathbb{D}}\left(1-|z|^{2}\right)\left|f^{\prime}(z)\right|<\infty .
$$

The mapping $f \mapsto \beta_{f}$ is a semi-norm on the space $\mathcal{B}$ of Bloch functions, called the Bloch space. Under the norm

$$
\|f\|_{\mathcal{B}}=|f(0)|+\beta_{f},
$$

the Bloch space is a Banach space. The little Bloch space $\mathcal{B}_{0}$ is the closed subspace of $\mathcal{B}$ consisting of functions $f \in \mathcal{B}$ satisfying

$$
\lim _{|z| \rightarrow 1}\left(1-|z|^{2}\right)\left|f^{\prime}(z)\right|=0 .
$$

For further treatment of Bloch functions and the Bloch space see [1, 3, 17].

Suppose $\psi$ is a fixed analytic function on $\mathbb{D}$. The multiplication operator on the Bloch space is defined as

$$
M_{\psi}(f)=\psi f
$$

(C) 2009 Australian Mathematical Society 0004-9727/2009 \$16.00 
In [2], Brown and Shields proved that $M_{\psi}$ is a bounded operator on the Bloch space if and only if $\psi \in H^{\infty}(\mathbb{D})$ and

$$
\left|\psi^{\prime}(z)\right|=O\left(\frac{1}{(1-|z|) \log (1 /(1-|z|))}\right) .
$$

This implies that

$$
\left(1-|z|^{2}\right)\left|\psi^{\prime}(z)\right|=O\left(\frac{1}{\log (1 /(1-|z|))}\right) \rightarrow 0
$$

as $|z| \rightarrow 1$. Thus $\psi \in \mathcal{B}_{0}$.

Suppose further that $\varphi$ is a fixed analytic self-map of $\mathbb{D}$. The weighted composition operator on the Bloch space is defined as

$$
W_{\psi, \varphi}(f)=\psi(f \circ \varphi)
$$

There is a reason for introducing the weighted composition operator at this point. The multiplication operator is a 'degenerate' weighted composition operator; if $\varphi$ is the identity map, then $W_{\psi, \varphi}=M_{\psi}$. Likewise, the composition operator on the Bloch space, defined as

$$
C_{\varphi}(f)=f \circ \varphi
$$

is a 'degenerate' weighted composition operator when $\psi$ is taken to be the constant function 1 .

In [16], Ohno and Zhao proved that $W_{\psi, \varphi}$ is a bounded operator on the Bloch space if and only if the following two properties are satisfied:

$$
\begin{gathered}
\sup _{z \in \mathbb{D}}\left(1-|z|^{2}\right)\left|\psi^{\prime}(z)\right| \log \frac{2}{1-|\varphi(z)|^{2}}<\infty, \\
\sup _{z \in \mathbb{D}} \frac{1-|z|^{2}}{1-|\varphi(z)|^{2}}|\psi(z)|\left|\varphi^{\prime}(z)\right|<\infty
\end{gathered}
$$

It can easily be proved that if $W_{\psi, \varphi}$ is bounded on the Bloch space, then $\psi$ is a Bloch function.

The characterization of the isometries is an open problem for most Banach spaces of analytic functions; the set of isometries, in most cases, is too large to study all at once. Even so, there are spaces for which the isometries are known. In [11], Forelli proved the isometries on the Hardy space $H^{p}$, for $p \neq 2$, are certain weighted composition operators. Also in [10], El-Gebeily and Wolfe completely characterized the isometries on the disk algebra $\mathcal{A}_{0}=H^{\infty}(\mathbb{D}) \cap C(\partial \mathbb{D})$. For most other spaces, a complete picture of the isometries is not known. The surjective isometries for the Bergman space $A^{p}$ (see [18]) and the weighted Bergman space $A_{\omega}^{p}$ (see [14]) are known.

The first attempt to study the isometries on the Bloch space was made by Cima and Wogen in [4]. They studied the isometries on the subspace of the Bloch space whose 
elements fix the origin. On this space, they showed that the surjective isometries are normalized compressions of composition operators induced by disk automorphisms. However, on the entire set of Bloch functions, a description of all isometries is still unknown. The current trend in the literature is to attack this problem one operator at a time.

To date, the only complete characterization of isometries on the Bloch space is those among the composition operators. Independently, two different descriptions have been developed. In [6], the second author identified the isometric composition operators in terms of the canonical factorization of the symbol. Martín and Vukotic [15] identified the isometric composition operators in terms of the hyperbolic derivative and cluster set of the symbol. The purpose of this paper is to further develop the understanding of the isometries on the Bloch space by characterizing the isometric multiplication operators. The authors are unaware of any such description of the isometric multiplication operators on the Bloch space in the literature.

1.1. Organization of the paper In Section 2, we determine estimates on the norm of the weighted composition operator $W_{\psi, \varphi}$ on the Bloch space. As a corollary, we deduce estimates on the norm of the multiplication operator $M_{\psi}$. In the case where $\psi \equiv 1$, the norm estimates agree with those derived by Xiong in [19] for the composition operator $C_{\varphi}$.

In Section 3, we prove that the symbols of isometric multiplication operators are precisely the constant functions of modulus 1 .

In Section 4, we show that the spectrum of the multiplication operator is the closure of the range of the symbol. From this, we deduce the spectra of the isometric multiplication operators to be single-element subsets of the unit circle.

In Section 5, we discuss the weighted composition operators whose symbols induce isometries individually and describe their spectrum.

\section{Norm estimates on weighted composition operators}

In this section we establish estimates on $\left\|W_{\psi, \varphi}\right\|$. In order to write these estimates in a succinct way, we introduce the following notation. Let $\psi \in \mathcal{B}$ and $\varphi: \mathbb{D} \rightarrow \mathbb{D}$ be analytic functions satisfying conditions (1.1) and (1.2). Let

$$
\begin{aligned}
\tau_{\psi, \varphi}^{\infty} \stackrel{\text { def }}{=} \sup _{z \in \mathbb{D}} \frac{1-|z|^{2}}{1-|\varphi(z)|^{2}}|\psi(z)|\left|\varphi^{\prime}(z)\right|, \\
\sigma_{\psi, \varphi}^{\infty} \stackrel{\text { def }}{=} \sup _{z \in \mathbb{D}} \frac{1}{2}\left(1-|z|^{2}\right)\left|\psi^{\prime}(z)\right| \log \frac{1+|\varphi(z)|}{1-|\varphi(z)|}
\end{aligned}
$$

which are both finite. In [19], Xiong defined

$$
\tau_{\varphi}^{\infty}=\sup _{z \in \mathbb{D}} \frac{1-|z|^{2}}{1-|\varphi(z)|^{2}}\left|\varphi^{\prime}(z)\right|
$$


to obtain an upper estimate on the norm of a composition operator on $\mathcal{B}$. Note that if $\psi \equiv 1$, then $\tau_{\psi, \varphi}^{\infty}=\tau_{\varphi}^{\infty}$. Also, if $\varphi$ is the identity map, then

$$
\sigma_{\psi, \varphi}^{\infty}=\sigma_{\psi}^{\infty} \stackrel{\text { def }}{=} \sup _{z \in \mathbb{D}} \frac{1}{2}\left(1-|z|^{2}\right)\left|\psi^{\prime}(z)\right| \log \frac{1+|z|}{1-|z|} .
$$

To determine an upper bound on $\left\|W_{\psi, \varphi}\right\|$ we use the following lemma, a proof of which can be found in [5] or [19].

LEMMA 2.1. If $f \in \mathcal{B}$, then

$$
|f(z)| \leq|f(0)|+\frac{1}{2} \beta_{f} \log \frac{1+|z|}{1-|z|},
$$

for all $z \in \mathbb{D}$.

With this lemma and the notation defined above, we are now able to establish an upper bound on $\left\|W_{\psi, \varphi}\right\|$.

THEOREM 2.2. Suppose that $\psi$ is an analytic function on $\mathbb{D}$ and $\varphi$ is an analytic self-map of $\mathbb{D}$ inducing a bounded weighted composition operator $W_{\psi, \varphi}$ on $\mathcal{B}$. Then

$$
\left\|W_{\psi, \varphi}\right\| \leq \max \left\{\|\psi\|_{\mathcal{B}}, \frac{1}{2}|\psi(0)| \log \frac{1+|\varphi(0)|}{1-|\varphi(0)|}+\tau_{\psi, \varphi}^{\infty}+\sigma_{\psi, \varphi}^{\infty}\right\} .
$$

Proof. Let $f \in \mathcal{B}$ such that $\|f\|_{\mathcal{B}}=1$. Then

$$
\begin{aligned}
\left\|W_{\psi, \varphi} f\right\|_{\mathcal{B}} \leq & |\psi(0)||f(\varphi(0))|+\sup _{z \in \mathbb{D}}\left(1-|z|^{2}\right)|\psi(z)|\left|f^{\prime}(\varphi(z))\right|\left|\varphi^{\prime}(z)\right| \\
& +\sup _{z \in \mathbb{D}}\left(1-|z|^{2}\right)\left|\psi^{\prime}(z)\right||f(\varphi(z))| \\
= & |\psi(0)||f(\varphi(0))|+\sup _{z \in \mathbb{D}} \frac{1-|z|^{2}}{1-|\varphi(z)|^{2}}|\psi(z)|\left|\varphi^{\prime}(z)\right|\left(1-|\varphi(z)|^{2}\right)\left|f^{\prime}(\varphi(z))\right| \\
& +\sup _{z \in \mathbb{D}}\left(1-|z|^{2}\right)\left|\psi^{\prime}(z)\right||f(\varphi(z))| \\
\leq & |\psi(0)||f(\varphi(0))|+\tau_{\psi, \varphi}^{\infty} \beta_{f}+\sup _{z \in \mathbb{D}}\left(1-|z|^{2}\right)\left|\psi^{\prime}(z)\right||f(\varphi(z))| .
\end{aligned}
$$

By Lemma 2.1,

$$
|f(\varphi(z))| \leq|f(0)|+\frac{1}{2} \beta_{f} \log \frac{1+|\varphi(z)|}{1-|\varphi(z)|}
$$

Thus

$$
\left\|W_{\psi, \varphi} f\right\|_{\mathcal{B}} \leq|\psi(0)||f(\varphi(0))|+\tau_{\psi, \varphi}^{\infty} \beta_{f}+\beta_{\psi}|f(0)|+\sigma_{\psi, \varphi}^{\infty} \beta_{f}
$$

Since

$$
|f(\varphi(0))| \leq|f(0)|+\frac{1}{2} \beta_{f} \log \frac{1+|\varphi(0)|}{1-|\varphi(0)|}
$$


and recalling that $|f(0)|=1-\beta_{f}$, we deduce that

$$
\begin{aligned}
\left\|W_{\psi, \varphi} f\right\|_{\mathcal{B}} & \leq\|\psi\|_{\mathcal{B}}|f(0)|+\left(\frac{1}{2}|\psi(0)| \log \frac{1+|\varphi(0)|}{1-|\varphi(0)|}+\tau_{\psi, \varphi}^{\infty}+\sigma_{\psi, \varphi}^{\infty}\right) \beta_{f} \\
& =\|\psi\|_{\mathcal{B}}+\left(\frac{1}{2}|\psi(0)| \log \frac{1+|\varphi(0)|}{1-|\varphi(0)|}+\tau_{\psi, \varphi}^{\infty}+\sigma_{\psi, \varphi}^{\infty}-\|\psi\|_{\mathcal{B}}\right) \beta_{f} .
\end{aligned}
$$

If

$$
\frac{1}{2}|\psi(0)| \log \frac{1+|\varphi(0)|}{1-|\varphi(0)|}+\tau_{\psi, \varphi}^{\infty}+\sigma_{\psi, \varphi}^{\infty} \leq\|\psi\|_{\mathcal{B}}
$$

then

$$
\left\|W_{\psi, \varphi} f\right\|_{\mathcal{B}} \leq\|\psi\|_{\mathcal{B}}
$$

If

$$
\frac{1}{2}|\psi(0)| \log \frac{1+|\varphi(0)|}{1-|\varphi(0)|}+\tau_{\psi, \varphi}^{\infty}+\sigma_{\psi, \varphi}^{\infty} \geq\|\psi\|_{\mathcal{B}},
$$

then

$$
\begin{aligned}
\left\|W_{\psi, \varphi} f\right\|_{\mathcal{B}} & \leq\|\psi\|_{\mathcal{B}}+\frac{1}{2}|\psi(0)| \log \frac{1+|\varphi(0)|}{1-|\varphi(0)|}+\tau_{\psi, \varphi}^{\infty}+\sigma_{\psi, \varphi}^{\infty}-\|\psi\|_{\mathcal{B}} \\
& =\frac{1}{2}|\psi(0)| \log \frac{1+|\varphi(0)|}{1-|\varphi(0)|}+\tau_{\psi, \varphi}^{\infty}+\sigma_{\psi, \varphi}^{\infty} .
\end{aligned}
$$

Therefore,

$$
\left\|W_{\psi, \varphi}\right\| \leq \max \left\{\|\psi\|_{\mathcal{B}}, \frac{1}{2}|\psi(0)| \log \frac{1+|\varphi(0)|}{1-|\varphi(0)|}+\tau_{\psi, \varphi}^{\infty}+\sigma_{\psi, \varphi}^{\infty}\right\},
$$

as desired.

To determine a lower bound on $\left\|W_{\psi, \varphi}\right\|$, we apply the appropriate test functions.

TheOrem 2.3. Suppose that $\psi$ is an analytic function on $\mathbb{D}$ and $\varphi$ is an analytic self-map of $\mathbb{D}$ inducing a bounded weighted composition operator $W_{\psi, \varphi}$ on $\mathcal{B}$. Then

$$
\left\|W_{\psi, \varphi}\right\| \geq \max \left\{\|\psi\|_{\mathcal{B}}, \frac{1}{2}|\psi(0)| \log \frac{1+|\varphi(0)|}{1-|\varphi(0)|}\right\} .
$$

PROOF. If we take the test function $f(z)=1$, then

$$
\left\|W_{\psi, \varphi}\right\| \geq\left\|W_{\psi, \varphi} f\right\|_{\mathcal{B}}=\|\psi\|_{\mathcal{B}} .
$$

If $\varphi(0)=0$, then (2.1) holds trivially. If $\varphi(0) \neq 0$, then write $\varphi(0)=|\varphi(0)| e^{i \theta}$, for some $\theta \in[0,2 \pi)$. Let

$$
f(z)=\frac{1}{2} \log \frac{1+e^{-i \theta} z}{1-e^{-i \theta} z}
$$


where Log denotes the principal branch of the logarithm. Then $f$ is a Bloch function with Bloch norm 1. Thus

$$
\left\|W_{\psi, \varphi}\right\| \geq\left\|W_{\psi, \varphi} f\right\|_{\mathcal{B}} \geq|\psi(0) f(\varphi(0))|=\frac{1}{2}|\psi(0)| \log \frac{1+|\varphi(0)|}{1-|\varphi(0)|} .
$$

Therefore,

$$
\left\|W_{\psi, \varphi}\right\| \geq \max \left\{\|\psi\|_{\mathcal{B}}, \frac{1}{2}|\psi(0)| \log \frac{1+|\varphi(0)|}{1-|\varphi(0)|}\right\}
$$

as desired.

REMARK 1 . By taking $\psi \equiv 1$, we deduce the estimates on the norm of $C_{\varphi}$ established in [19]:

$$
\max \left\{1, \frac{1}{2} \log \frac{1+|\varphi(0)|}{1-|\varphi(0)|}\right\} \leq\left\|C_{\varphi}\right\| \leq \max \left\{1, \frac{1}{2} \log \frac{1+|\varphi(0)|}{1-|\varphi(0)|}+\tau_{\varphi}^{\infty}\right\} .
$$

We now deduce estimates on the norm of the multiplication operator $M_{\psi}$ on $\mathcal{B}$.

COROllary 2.4. Suppose $\psi$ is an analytic function on $\mathbb{D}$ inducing a bounded multiplication operator $M_{\psi}$ on $\mathcal{B}$. Then

$$
\max \left\{\|\psi\|_{\mathcal{B}},\|\psi\|_{\infty}\right\} \leq\left\|M_{\psi}\right\| \leq \max \left\{\|\psi\|_{\mathcal{B}},\|\psi\|_{\infty}+\sigma_{\psi}^{\infty}\right\} .
$$

In particular, if $\psi(0)=0$, then

$$
\|\psi\|_{\infty} \leq\left\|M_{\psi}\right\| \leq\|\psi\|_{\infty}+\sigma_{\psi}^{\infty} .
$$

ProOF. By taking $\varphi$ to be the identity map, $\tau_{\psi, \varphi}^{\infty}=\|\psi\|_{\infty}$ and $\sigma_{\psi, \varphi}^{\infty}=\sigma_{\psi}^{\infty}$. Thus,

$$
\|\psi\|_{\mathcal{B}} \leq\left\|M_{\psi}\right\| \leq \max \left\{\|\psi\|_{\mathcal{B}},\|\psi\|_{\infty}+\sigma_{\psi}^{\infty}\right\} .
$$

Furthermore, we have $\|\psi\|_{\infty} \leq\left\|M_{\psi}\right\|$. Indeed, this is true for a bounded multiplication operator on any functional Banach space (see [9, Lemma 11]). Therefore,

$$
\max \left\{\|\psi\|_{\mathcal{B}},\|\psi\|_{\infty}\right\} \leq\left\|M_{\psi}\right\| \leq \max \left\{\|\psi\|_{\mathcal{B}},\|\psi\|_{\infty}+\sigma_{\psi}^{\infty}\right\},
$$

as desired. The conclusion for the case $\psi(0)=0$ follows from the fact that by the Schwarz-Pick lemma, for a bounded function $\psi$ on $\mathbb{D}$,

$$
\left(1-|z|^{2}\right) \frac{\left|\psi^{\prime}(z)\right|}{\|\psi\|_{\infty}} \leq 1-\frac{|\psi(z)|^{2}}{\|\psi\|_{\infty}^{2}}
$$

for all $z$ in $\mathbb{D}$, so that $\|\psi\|_{\mathcal{B}}=\beta_{\psi} \leq\|\psi\|_{\infty}$. 
Open Question. If $M_{\psi}$ is bounded on $\mathcal{B}$, does the inequality $\|\psi\|_{\mathcal{B}} \leq\|\psi\|_{\infty}+\sigma_{\psi}^{\infty}$ hold? As mentioned above, it does hold if $\psi$ fixes the origin. It also holds in the case of $\psi$ being an automorphism of $\mathbb{D}$. Indeed, suppose that $\psi \in \operatorname{Aut}(\mathbb{D})$ with $\psi(0) \neq 0$. Then

$$
\psi(z)=\eta \frac{a-z}{1-\bar{a} z}
$$

where $0 \neq a \in \mathbb{D}$ and $|\eta|=1$. Thus, $\|\psi\|_{\infty}=1$ and $\beta_{\psi}=\left(1-|a|^{2}\right)\left|\psi^{\prime}(a)\right|=1$, so $\|\psi\|_{\mathcal{B}}=|a|+1$. Furthermore,

$$
\begin{aligned}
\sigma_{\psi}^{\infty} & =\sup _{z \in \mathbb{D}}\left(1-|z|^{2}\right)\left|\psi^{\prime}(z)\right| \frac{1}{2} \log \frac{1+|z|}{1-|z|} \\
& \geq\left(1-|a|^{2}\right)\left|\psi^{\prime}(a)\right| \frac{1}{2} \log \frac{1+|a|}{1-|a|} \\
& =\frac{1}{2} \log \frac{1+|a|}{1-|a|}>|a|
\end{aligned}
$$

since, for $|a|<1$,

$$
\frac{1}{2} \log \frac{1+|a|}{1-|a|}=\sum_{n=0}^{\infty} \frac{|a|^{2 n+1}}{2 n+1} .
$$

Therefore $\|\psi\|_{\mathcal{B}} \leq\|\psi\|_{\infty}+\sigma_{\psi}^{\infty}$.

\section{Characterization of isometric multiplication operators}

This section is devoted to the identification of the symbols of the isometric multiplication operators on the Bloch space. We first establish necessary conditions for $\psi$ to induce an isometric multiplication operator on $\mathcal{B}$.

LEMMA 3.1. Let $\psi$ be the symbol of an isometric multiplication operator on $\mathcal{B}$. Then $\|\psi\|_{\infty} \leq 1$ and $\left\|\psi^{n}\right\|_{\mathcal{B}}=1$ for all $n \in \mathbb{N}$.

PROOF. By the lower estimate in Corollary 2.4, we obtain $\|\psi\|_{\infty} \leq\left\|M_{\psi}\right\|=1$. Choosing the test function $g \equiv 1$ gives

$$
1=\|g\|_{\mathcal{B}}=\left\|M_{\psi} g\right\|_{\mathcal{B}}=\|\psi\|_{\mathcal{B}}
$$

Thus

$$
\left\|\psi^{2}\right\|_{\mathcal{B}}=\left\|M_{\psi}(\psi)\right\|_{\mathcal{B}}=\|\psi\|_{\mathcal{B}}=1
$$

The conclusion follows by induction.

The next result is more general, and with it we can prove the main theorem.

LEMMA 3.2. If $\psi \in H^{\infty}(\mathbb{D})$ such that $\|\psi\|_{\infty} \leq 1$ and $\psi(0)=0$, then $\left\|\psi^{n}\right\|_{\mathcal{B}}<1$ for all $n \geq 2$. 
PROOF. By the Schwarz-Pick lemma, for all $n \in \mathbb{N}, n \geq 2$,

$$
\begin{aligned}
\beta_{\psi^{n}} & =\sup _{z \in \mathbb{D}}\left(1-|z|^{2}\right) n|\psi(z)|^{n-1}\left|\psi^{\prime}(z)\right| \\
& \leq \sup _{z \in \mathbb{D}} n\left(1-|\psi(z)|^{2}\right)|\psi(z)|^{n-1} \\
& \leq n \max _{x \in[0,1]}\left(x^{n-1}-x^{n+1}\right) \\
& =\frac{2 n}{n+1}\left(\frac{n-1}{n+1}\right)^{(n-1) / 2}=\frac{2 n}{n-1}\left(\frac{n-1}{n+1}\right)^{(n+1) / 2} .
\end{aligned}
$$

For $x$ and $a$ positive real numbers and $m$ a real number greater than 1, we have $(x+a)^{m}>x^{m}+a m x^{m-1}$. Thus, for $n \geq 2$,

$$
(n+1)^{(n+1) / 2}>(n-1)^{(n+1) / 2}+2\left(\frac{n+1}{2}\right)(n-1)^{(n-1) / 2}=2 n(n-1)^{(n-1) / 2} .
$$

So

$$
\frac{2 n}{n+1}\left(\frac{n-1}{n+1}\right)^{(n-1) / 2}=\frac{2 n(n-1)^{(n-1) / 2}}{(n+1)^{(n+1) / 2}}<1 .
$$

Hence $\left\|\psi^{n}\right\|_{\mathcal{B}}=\beta_{\psi^{n}}<1$ for $n \geq 2$.

COROLlary 3.3. If $\psi$ is the symbol of an isometric multiplication operator on $\mathcal{B}$, then $\psi$ does not fix the origin.

PROOF. Arguing by contradiction, assume that $\psi(0)=0$. By Lemma 3.1, $\|\psi\|_{\infty} \leq 1$ and $\left\|\psi^{2}\right\|_{\mathcal{B}}=1$. However, $\left\|\psi^{2}\right\|_{\mathcal{B}}<1$ by Lemma 3.2.

Lemma 3.4. Suppose that $\psi \in H^{\infty}(\mathbb{D})$ such that $\|\psi\|_{\infty} \leq 1$ and the map $g(z)=z \psi(z)$ has Bloch norm 1. Then either $\psi$ is a constant of modulus 1 , or $\psi$ has infinitely many zeros $\left\{a_{n}\right\}$ in $\mathbb{D}$ such that

$$
\beta_{\psi}=\limsup _{n \rightarrow \infty}\left(1-\left|a_{n}\right|^{2}\right)\left|\psi^{\prime}\left(a_{n}\right)\right|=1
$$

In the latter case, if $\|\psi\|_{\mathcal{B}}=1$, then $\psi(0)=0$.

PROOF. Assume that $\psi$ is not a constant of modulus 1 . Note that the function $g$ maps $\mathbb{D}$ into itself, fixes the origin, and has Bloch norm 1 . Then by [6, Theorem 3], there exists an infinite sequence $\left\{a_{n}\right\}$ in $\mathbb{D}$ such that $\psi\left(a_{n}\right)=0$ and

$$
\limsup _{n \rightarrow \infty}\left(1-\left|a_{n}\right|^{2}\right)\left|g^{\prime}\left(a_{n}\right)\right|=1 \text {. }
$$

Since $g^{\prime}\left(a_{n}\right)=\psi\left(a_{n}\right)+a_{n} \psi^{\prime}\left(a_{n}\right)=a_{n} \psi^{\prime}\left(a_{n}\right)$, we deduce that

$$
\limsup _{n \rightarrow \infty}\left(1-\left|a_{n}\right|^{2}\right)\left|a_{n}\right|\left|\psi^{\prime}\left(a_{n}\right)\right|=1 \text {. }
$$


By assumption $\psi$ is not a constant function, and so $\left|a_{n}\right| \rightarrow 1$ as $n \rightarrow \infty$. Thus

$$
\beta_{\psi}=\limsup _{n \rightarrow \infty}\left(1-\left|a_{n}\right|^{2}\right)\left|\psi^{\prime}\left(a_{n}\right)\right|=1 .
$$

The conclusion for the case $\|\psi\|_{\mathcal{B}}=1$ follows at once.

We now prove the main theorem of this section.

THEOREM 3.5. The multiplication operator $M_{\psi}$ is an isometry on $\mathcal{B}$ if and only if $\psi$ is a constant function of modulus 1 .

PROOF. Clearly, if $\psi$ is a constant function of modulus 1 then $M_{\psi}$ is an isometry on $\mathcal{B}$. Conversely, suppose that $M_{\psi}$ is an isometry on $\mathcal{B}$ and assume that $\psi$ is not a constant function of modulus 1 . Then by Lemma 3.1, $\|\psi\|_{\infty} \leq 1$ and $\|\psi\|_{\mathcal{B}}=1$. Also, for $g(z)=z \psi(z),\|g\|_{\mathcal{B}}=\left\|M_{\psi}(\mathrm{id})\right\|_{\mathcal{B}}=\|\mathrm{id}\|_{\mathcal{B}}=1$, where id is the identity map on $\mathbb{D}$. Then by Lemma 3.4, $\psi(0)=0$, contradicting Corollary 3.3. Therefore, if $M_{\psi}$ is an isometry on $\mathcal{B}$, then $\psi$ must be a constant function of modulus 1 .

\section{Characterization of the spectra of multiplication operators}

We now turn our attention to the spectrum of a multiplication operator. Recall that the resolvent of a bounded linear operator $T$ on a complex Banach space $E$ is defined as

$$
\rho(T)=\{\lambda \in \mathbb{C} \mid T-\lambda I \text { is invertible }\},
$$

where $I$ is the identity operator. The spectrum of $T$ is defined as $\sigma(T)=\mathbb{C} \backslash \rho(T)$. The approximate point spectrum, a subset of the spectrum, is defined as

$$
\sigma_{a p}(T)=\{\lambda \in \mathbb{C} \mid T-\lambda I \text { is not bounded below }\},
$$

that is, for every $M>0$, there exists $x \in E$ such that $\|T x\|<M\|x\|$.

The spectrum is a nonempty compact subset of the closed disk centered at the origin of radius $\|T\|$. In particular, the spectrum of an isometry is contained in $\overline{\mathbb{D}}$. Furthermore, the boundary $\partial \sigma(T)$ of $\sigma(T)$ is a subset of $\sigma_{a p}(T)$ (see [7, Proposition 6.7]).

THEOREM 4.1. Let $\psi$ be the symbol of a bounded multiplication operator $M_{\psi}$ on $\mathcal{B}$. Then $\sigma\left(M_{\psi}\right)=\overline{\psi(\mathbb{D})}$.

PROOF. For $\lambda \in \mathbb{C}$, the operator $M_{\psi}-\lambda I$ can be rewritten as $M_{\psi-\lambda}$. Thus $\lambda \in \sigma\left(M_{\psi}\right)$ if and only if $M_{\psi-\lambda}$ is not invertible. Clearly, if $M_{\psi-\lambda}^{-1}$ exists, it is the multiplication operator $M_{(\psi-\lambda)^{-1}}$.

Let $\lambda \in \psi(\mathbb{D})$. Then there exists $z_{0} \in \mathbb{D}$ such that $\psi\left(z_{0}\right)=\lambda$. So $(\psi-\lambda)^{-1}$ has a pole at $z_{0}$, which means that $M_{(\psi-\lambda)^{-1}}$ is not a well-defined operator. Thus $M_{\psi-\lambda}$ is not invertible. This implies that $\overline{\psi(\mathbb{D})} \subseteq \sigma\left(M_{\psi}\right)$. 
Suppose that $\lambda \notin \overline{\psi(\mathbb{D})}$. Then $|\psi-\lambda|$ is bounded away from 0 by some positive constant $c$. Thus the modulus of the function $g(z)=1 /(\psi(z)-\lambda)$ is in $H^{\infty}(\mathbb{D})$. In addition,

$$
\left|g^{\prime}(z)\right|=\frac{\left|\psi^{\prime}(z)\right|}{|\psi(z)-\lambda|^{2}} \leq \frac{1}{c^{2}}\left|\psi^{\prime}(z)\right|=O\left(\frac{1}{(1-|z|) \log (1 / 1-|z|)}\right) .
$$

So $M_{g}=M_{(\psi-\lambda)^{-1}}$ is a bounded operator on $\mathcal{B}$. Thus $\lambda \notin \sigma\left(M_{\psi}\right)$. Therefore $\sigma\left(M_{\psi}\right)=\overline{\psi(\mathbb{D})}$.

This result is not surprising since it also holds for the space of continuous, realvalued functions on a closed interval. A similar result holds for $L^{2}(\mu), \mu$ a probability measure. Specifically, for $\psi \in L^{\infty}(\mu)$, the spectrum of $M_{\psi}$ on $L^{2}(\mu)$ is the essential range of $\psi$, that is, the set of $\lambda \in \mathbb{C}$ such that the preimage under $\psi$ of every neighborhood of $\lambda$ has positive measure [8]. As an immediate consequence of Theorems 3.5 and 4.1, we obtain the following result.

Corollary 4.2. Let $M_{\psi}$ be an isometric multiplication operator on the Bloch space. Then $\sigma\left(M_{\psi}\right)=\{\eta\}$, where $\eta$ is the unimodular constant value of $\psi$.

\section{A further glimpse at weighted composition operators}

Our focus returns to weighted composition operators, and isometries amongst them. Let $\mathcal{I}_{W}$ be the set of weighted composition operators $W_{\psi, \varphi}$ such that $\psi$ induces an isometric multiplication operator and $\varphi$ induces an isometric composition operator. Clearly the set of isometric weighted composition operators contains $\mathcal{I}_{W}$.

ObSeRvation 5.1. In [6, Theorem 2], it was shown that $\varphi$ induces an isometric composition operator on $\mathcal{B}$ if and only if $\varphi(0)=0$ and $\beta_{\varphi}=1$. In particular, [6, Corollary 2] proved that either $\varphi$ is a rotation or the zero set of $\varphi$ forms an infinite sequence $\left\{a_{n}\right\}$ in $\mathbb{D}$ such that

$$
\limsup _{n \rightarrow \infty}\left(1-\left|a_{n}\right|^{2}\right)\left|\varphi^{\prime}\left(a_{n}\right)\right|=1 .
$$

Notice that the class $\mathfrak{I}$ of such functions $\varphi$ is very large. Indeed, $\varphi \in \mathfrak{I}$ if and only if $\varphi=g B$ where $g$ is a nonvanishing analytic function of $\mathbb{D}$ into $\overline{\mathbb{D}}$, and $B$ is a Blaschke product whose zeros form an infinite sequence $\left\{a_{n}\right\}$ containing 0 and an infinite subsequence $\left\{a_{n_{j}}\right\}$ such that $\left|g\left(a_{n_{j}}\right)\right| \rightarrow 1$ and

$$
\lim _{j \rightarrow \infty} \prod_{k \neq n_{j}}\left|\frac{a_{n_{j}}-a_{k}}{1-\overline{a_{n_{j}}} a_{k}}\right|=1 \text {. }
$$

The Blaschke products whose zeros satisfy (5.1) include the thin Blaschke products. For more on this topic, see [12, 13].

OPEN QUESTION. Are there any isometric weighted composition operators whose symbols do not induce isometric multiplication and composition operators individually? 
If the answer to the above question is negative, then $\mathcal{I}_{W}$ is exactly the set of isometric weighted composition operators on the Bloch space and we have a complete characterization of their symbols.

At this time, we describe the spectrum of isometric composition operators, which with Corollary 4.2 will be used to describe the spectrum of the elements of $\mathcal{I}_{W}$. To do so, we need an important result about the spectrum of an isometry on a general Banach space. The following proposition is found (in a slightly different form) as an exercise in [7], and we provide a proof for completeness.

Proposition 5.2. Let $E$ be a complex Banach space and suppose that $T: E \rightarrow E$ is an isometry. If $T$ is invertible, then $\sigma(T) \subseteq \partial \mathbb{D}$. If $T$ is not invertible, then $\sigma(T)=\overline{\mathbb{D}}$.

ProOf. Suppose that $T$ is an invertible isometry on $E$. Then $0 \notin \sigma(T)$, and so the function $z \mapsto z^{-1}$ is analytic in some neighborhood of $\sigma(T)$. By the spectral mapping theorem (see [7, p. 204]), $\sigma(f \circ T)=f(\sigma(T))$, and so

$$
\sigma\left(T^{-1}\right)=\sigma(T)^{-1}=\left\{\lambda^{-1} \mid \lambda \in \sigma(T)\right\} .
$$

Since $T^{-1}$ exists and is an isometry, $\sigma\left(T^{-1}\right) \subseteq \overline{\mathbb{D}}$. Therefore, $\sigma(T) \subseteq \partial \mathbb{D}$.

Next, suppose that $T$ is not invertible. In order to prove that $\sigma(T)=\overline{\mathbb{D}}$, it suffices to show that $\mathbb{D} \subseteq \sigma(T)$. For $\lambda \in \mathbb{D}, T-\lambda I$ is bounded below by $1-|\lambda|$. Thus, $\lambda \notin \sigma_{a p}(T)$. We deduce that $\partial \sigma(T) \subseteq \sigma_{a p}(T) \subseteq \partial \mathbb{D}$.

Assume that $\lambda \in \overline{\mathbb{D}} \cap \rho(T)$. Note that $\lambda \notin \partial \sigma(T)$ since $\partial \sigma(T)=\sigma(T) \cap \overline{\rho(T)}$. Consider $\Gamma=\{t \lambda \mid t \in[0, \infty)\}$, the radial line through $\lambda$. Since $\sigma(T)$ is closed and $0 \in \sigma(T)$, there exists $t \in[0,1)$ such that $t \lambda \in \partial \sigma(T)$. This contradicts the fact that $\partial \sigma(T) \subseteq \partial \mathbb{D}$. Consequently, $\mathbb{D} \subseteq \sigma(T)$.

For a complex number $\zeta$ of modulus 1, define the order of $\zeta$, denoted by $\operatorname{ord}(\zeta)$, to be the smallest $n \in \mathbb{N}$ such that $\zeta^{n}=1$. If no such $n$ exists, we say that $\zeta$ has infinite order, and write $\operatorname{ord}(\zeta)=\infty$.

THEOREM 5.3. Suppose that $\varphi$ induces an isometric composition operator on $\mathcal{B}$. If $\varphi$ is not a rotation, then $\sigma\left(C_{\varphi}\right)=\overline{\mathbb{D}}$. If $\varphi(z)=\zeta z$ with $|\zeta|=1$, then

$$
\sigma\left(C_{\varphi}\right)= \begin{cases}\partial \mathbb{D} & \text { if } \operatorname{ord}(\zeta)=\infty \\ \langle\zeta\rangle & \text { if } \operatorname{ord}(\zeta)<\infty\end{cases}
$$

where $\langle\zeta\rangle$ is the cyclic group generated by $\zeta$.

Proof. Assume that $\varphi$ is not a rotation. Then by Proposition 5.2 it suffices to show that $C_{\varphi}$ is not invertible. Since $C_{\varphi}$ is an isometry it is necessarily injective. Thus, we shall show that $C_{\varphi}$ is not surjective. Arguing by contradiction, assume that $C_{\varphi}$ is surjective. Since $\varphi$ is not a rotation, by Observation $5.1 \varphi$ has infinitely many zeros. Let $a$ and $a^{\prime}$ be distinct zeros of $\varphi$.

Define $h(z)=z-a$, and note that $h$ is a nonzero Bloch function. Since $C_{\varphi}$ is assumed to be surjective, there exists a Bloch function $f$ such that $h=f \circ \varphi$. 
On the one hand, $f(0)=f\left(\varphi\left(a^{\prime}\right)\right)=h\left(a^{\prime}\right) \neq 0$. On the other hand, $f(0)=f(\varphi(a))$ $=h(a)=0$, a contradiction. Thus, $C_{\varphi}$ is not surjective. Therefore $\sigma\left(C_{\varphi}\right)=\overline{\mathbb{D}}$.

Now suppose that $\varphi(z)=\zeta z$ with $|\zeta|=1$. Then $\varphi^{-1}(z)=1 / \zeta z$ and $C_{\varphi}^{-1}=C_{\varphi^{-1}}$. So by Proposition 5.2, $\sigma\left(C_{\varphi}\right) \subseteq \partial \mathbb{D}$.

Let $G=\langle\zeta\rangle=\left\{\zeta^{k} \mid k \in \mathbb{N} \cup\{0\}\right\}$. Note that $G \subseteq \partial \mathbb{D}$. Consider the Bloch function $f(z)=z^{k}$ for $k \in \mathbb{N} \cup\{0\}$. Then

$$
\left(C_{\varphi} f\right)(z)=\zeta^{k} z^{k}=\zeta^{k} f(z)
$$

Thus $\zeta^{k}$ is an eigenvalue of $C_{\varphi}$ with corresponding eigenfunction $f$. So $G \subseteq \sigma\left(C_{\varphi}\right)$.

If the order of $\zeta$ is infinite, then $G$ is dense in $\partial \mathbb{D}$. Since the spectrum is closed, we have $\partial \mathbb{D}=\bar{G} \subseteq \sigma\left(C_{\varphi}\right)$. Thus $\sigma\left(C_{\varphi}\right)=\partial \mathbb{D}$.

Now suppose that $\operatorname{ord}(\zeta)=n<\infty$. Then $G=\left\{\zeta^{k} \mid k=1, \ldots, n\right\}$. We wish to show that $\sigma\left(C_{\varphi}\right) \subseteq G$. Let $\mu \in \partial \mathbb{D} \backslash G$. We shall show that $C_{\varphi}-\mu I$ is invertible by proving that for every $g \in \mathcal{B}$, there exists a unique $f \in \mathcal{B}$ such that $f \circ \varphi-\mu f=g$. Since $\operatorname{ord}(\zeta)=n$, then

$$
\varphi^{(n)}(z) \stackrel{\text { def }}{=}(\underbrace{\varphi \circ \cdots \circ \varphi}_{n \text { times }})(z)=\zeta^{n} z=z .
$$

By repeated application of $\varphi$, we can form the following system of equations:

$$
\begin{array}{llll}
f(\varphi(z)) & -\mu f(z) & = & g(z) \\
f\left(\varphi^{(2)}(z)\right) & -\mu f(\varphi(z)) & = & g(\varphi(z)) \\
& \vdots & \vdots & \\
f(z) & -\mu f\left(\varphi^{(n-1)}(z)\right) & = & g\left(\varphi^{(n-1)}(z)\right) .
\end{array}
$$

Equivalently, (5.2) can be posed as the matrix equation $A x=b$ where

$$
\begin{gathered}
A=\left[\begin{array}{cccccc}
-\mu & 1 & 0 & 0 & \ldots & 0 \\
0 & -\mu & 1 & 0 & \ldots & 0 \\
\vdots & 0 & \ddots & \ddots & & \vdots \\
\vdots & & \ddots & \ddots & \ddots & \vdots \\
0 & & & \ddots & \ddots & 1 \\
1 & 0 & \ldots & \cdots & 0 & -\mu
\end{array}\right], \quad x=\left[\begin{array}{c}
f(z) \\
g(z) \\
g(\varphi(z)) \\
\vdots \\
\vdots \\
\vdots \\
g\left(\varphi^{(n-2)}(z)\right) \\
g\left(\varphi^{(n-1)}(z)\right)
\end{array}\right] \\
b=\left[\begin{array}{c}
(n-2)(z)) \\
f\left(\varphi^{(n-1)}(z)\right)
\end{array}\right]
\end{gathered}
$$


Direct calculation shows that $\operatorname{det}(A)=(-1)^{n}\left(\mu^{n}-1\right) \neq 0$, since $\mu \notin G$. Thus, $C_{\varphi}-\mu I$ is invertible. For $\mu \notin G$, the unique solution $f$ of (5.2) is a (finite) linear combination of the functions $g \circ \varphi^{(j-1)}$, for $j=1, \ldots, n$, each of which is Bloch. Thus $f$ is Bloch. Therefore $\sigma\left(C_{\varphi}\right)=G$.

THEOREM 5.4. Let $\psi$ induce an isometric multiplication operator (so that $\psi$ is a constant $\eta$ of modulus 1) and $\varphi$ induce an isometric composition operator. If $\varphi$ is not a rotation, then $\sigma\left(W_{\psi, \varphi}\right)=\overline{\mathbb{D}}$. If $\varphi=\zeta z$ for $|\zeta|=1$, then

$$
\sigma\left(W_{\psi, \varphi}\right)= \begin{cases}\partial \mathbb{D} & \text { if } \operatorname{ord}(\zeta)=\infty \\ \eta\langle\zeta\rangle & \text { if } \operatorname{ord}(\zeta)<\infty\end{cases}
$$

where $\eta\langle\zeta\rangle=\left\{\eta \zeta^{k} \mid k=1, \ldots, n\right\}$.

PROOF. Observe that $W_{\psi, \varphi}=\eta C_{\varphi}$ and, for $\lambda \in \mathbb{C}, W_{\psi, \varphi}-\lambda I=\eta\left(C_{\varphi}-\lambda \bar{\eta} I\right)$. Thus, $W_{\psi, \varphi}-\lambda I$ is not invertible if and only if $C_{\varphi}-\lambda \bar{\eta} I$ is not invertible. Thus $\lambda \in \sigma\left(W_{\psi, \varphi}\right)$ if and only if $\lambda \bar{\eta} \in \sigma\left(C_{\varphi}\right)$. The result follows immediately from Theorem 5.3.

\section{References}

[1] J. M. Anderson, J. Clunie and Ch. Pommerenke, 'On Bloch functions and normal functions', J. Reine Angew. Math. 279 (1974), 12-37.

[2] L. Brown and A. L. Shields, 'Multipliers and cyclic vectors in the Bloch space', Michigan Math. J. 38 (1991), 141-146.

[3] J. Cima, 'The basic properties of Bloch functions', Int. J. Math. Math. Sci. 3 (1979), 369-413.

[4] J. Cima and W. Wogen, 'On isometries of the Bloch space', Illinois J. Math. 24 (1980), 313-316.

[5] F. Colonna, 'Extreme points of a convex set of Bloch functions', Seminars in Complex Analysis and Geometry, Sem. Conf. 4 (1988), 23-59.

[6] 'Characterisation of the isometric composition operators on the Bloch space', Bull. Aust. Math. Soc. 72 (2005), 283-290.

[7] J. B. Conway, A Course in Functional Analysis, 2nd edn (Springer, New York, 1990).

[8] R. G. Douglas, Banach Algebra Techniques in Operator Theory, 2nd edn (Springer, New York, 1998).

[9] P. L. Duren, B. W. Romberg and A. L. Shields, 'Linear functionals on $H^{p}$ spaces with $0<p<1$ ', J. Reine Angew. Math. 238 (1969), 32-60.

[10] M. El-Gebeily and J. Wolfe, 'Isometries of the disc algebra', Proc. Amer. Math. Soc. 93 (1985), 697-702.

[11] F. Forelli, 'The isometries on $H^{p}$, Canad. J. Math. 16 (1964), 721-728.

[12] P. Gorkin and R. Mortini, 'Universal Blaschke products', Proc. Cambridge Philos. Soc. 136 (2004), 175-184.

[13] H. Hedenmalm, 'Thin interpolating sequences and three algebras of bounded functions', Proc. Amer. Math. Soc. 99 (1987), 489-495.

[14] C. J. Kolaski, 'Isometries of weighted Bergman spaces', Canad. J. Math 34 (1982), 910-915.

[15] M. J. Martín and D. Vukotić, 'Isometries of the Bloch space among the composition operators', Bull. London Math. Soc. 39 (2007), 151-155.

[16] S. Ohno and R. Zhao, 'Weighted composition operators on the Bloch space', Bull. Aust. Math. Soc. 63 (2001), 177-185.

[17] Ch. Pommerenke, 'On Bloch functions', J. London Math. Soc. 2 (1970), 689-695. 
[18] W. Rudin, ' $L^{p}$-isometries and equimeasurability', Indiana Univ. Math. J. 25 (1976), 215-228.

[19] C. Xiong, 'Norm of composition operators on the Bloch space', Bull. Aust. Math. Soc. 70 (2004), 293-299.

ROBERT F. ALLEN, Department of Mathematical Sciences,

George Mason University, 4400 University Drive, Fairfax, VA 22030, USA

e-mail: rallen2@gmu.edu

FLAVIA COLONNA, Department of Mathematical Sciences,

George Mason University, 4400 University Drive, Fairfax, VA 22030, USA

e-mail: fcolonna@gmu.edu 